\title{
Implementing the future hospital
}

\section{About this section}

For the last 4 years, this part of the Future Healthcare Journal has been the place to find regular overview updates on progress made by the Future Hospital Programme of the Royal College of Physicians, together with its partners, in realising the vision of the Future Hospital Commission.

As outlined in this article, the Future Hospital Programme has now concluded. However, much of its work is being carried on by the RCP Quality Improvement Programme, which can be contacted on RCPQI@rcplondon.ac.uk.

Follow the Quality Improvement Programme on Twitter: @RCP_QI

\section{Synopsis}

The final report of the RCP's Future Hospital Programme has shown that implementing the principles of Future Hospital: Caring for medical patients ${ }^{1}$ can deliver not only improved patient care and experience, but also cost, time and resource savings. Other benefits included staff retention, better morale and teamworking, and improvements in training and learning. Here, the Future Hospital officers (in post from 2014 to 2017) share the key messages from the report as well as the findings identified in the independent evaluation of the FHP undertaken by the University of Liverpool.

\section{Introduction}

The Royal College of Physicians (RCP) established the Future Hospital Programme (FHP) in 2013 in response to recommendations of the seminal Future Hospital Commission (FHC) report ${ }^{1}$ which described a bold new model of patientcentred care, underpinned by a core set of principles and new approaches to leadership and training. The FHP also demonstrated the RCP's commitment to being part of the wider solution to the challenges being faced by the NHS.

In November 2017, the RCP published the FHP's final report, Delivering the future hospital. The report included independent evaluations of the FHP overall and the chief registrar leadership and training programme. The report described a new model of patient-centred care underpinned by a core set of principles, including:

> care should come to the acutely ill patient, rather than the patient being moved around the hospital

$>$ care should be seamlessly provided across the hospital and community sectors.

Current pressures mean it is more important than ever to design and deliver services based on the needs of patients and carers. Delivering the Future Hospital is testimony to what can be achieved. - Elisabeth Davies, chair, Patient and Carer Network

\section{Championing patient experience}

The FHP has demonstrated that a patient-centred approach to improving services can help deliver better care for patients by more motivated, engaged staff. The FHP was a unique, comprehensive programme of activity which included: eight Future Hospital development sites (selected, supported local healthcare project teams); a pilot of the role of chief registrar (a senior clinical leadership role for experience trainee doctors); and other workstreams relating to person-centred care, young adults and adolescents and integrated care. The FHP:

> championed patient experience and patient-centred care throughout, by involving patients, carers and the public in service redesign and delivering from the outset

> embedded in clinical practice the FHC 'blueprint' and its 11 principles of patient care

> applied a standardised approach to measuring the impact of new ways of working through quality improvement methodology

> supported development sites to improve front-line services within existing local resources with no additional transformational funding

> developed future clinical leaders through a bespoke leadership and management programme: the chief registrar scheme

$>$ used the expertise and influence of a medical royal college to support improvements to patient care

> commissioned independent evaluations by the University of Liverpool (of FHP as a whole) (see Box 1) and University of Birmingham (of the chief registrar scheme).

\section{Successes}

Across the eight development sites, proven outcomes included direct benefits for patients and the NHS, including reduced admissions and readmissions, decreased length of stay, quicker patient assessment, improved patient feedback and measurement of patient experience, and improved patient involvement. Other indirect benefits included better staff retention, and improvements in training.

\section{Bringing care to the patient}

> Patients on surgical pathways who had access to acute physicians and geriatricians used ambulatory care more and had shorter hospital lengths of stay.

> Patients receiving comprehensive geriatric assessment from a specialist multidisciplinary team tended to have a shorter length of stay in hospital.

Providing care seamlessly across the hospital and community sectors

> Patients with respiratory illness experienced longer intervals between emergency admissions once specialist services were integrated. 
Box 1. An independent, external evaluation by the University of Liverpool

The RCP commissioned an independent evaluation of the FHP by the Department of Health Services Research and School of Management at the University of Liverpool. There were two primary aims for the evaluation: first, to assess the impact of the FHP on patients, professionals and clinical processes, and second, to assess whether the implementation of the FHP model is sustainable and widely applicable.

\section{Background}

Following the Future Hospital Commission (FHC) report, the RCP set up the Future Hospital Programme (FHP) to put these visions into practice. The Future Hospital Programme had various foci of activity, this included providing support to eight development sites to implement projects surrounding the FHC report principals and engaging the health care community. The RCP sought an external group to undertake an independent evaluation. The full report presents the findings of that external evaluation (www. rcplondon.ac.uk/delivering-the-future-hospital).

\section{Methods}

A mixed methods approach was used. Opinions about the FHP were sought from four main sources: the development site teams, the patient representatives from the development sites, personnel from the RCP both directly and indirectly involved with the programme, and the wider college membership. Activities involved focus groups, one-to-one interviews, a comprehensive documents review and web-based surveys.

\section{Key findings}

This evaluation has confirmed that the programme has had many successes and brought about real change; developed QI capacity directly within teams and more widely across the RCP; and demonstrated it is possible for the vision of the Future Hospital Commission to be delivered within real world environments.

It has demonstrated that colleges are well placed to lead on quality improvement work. The programme links well to future plans for the Quality Improvement Hub in the RCP, as well as the chief registrar scheme and the web-based 'Tell Us Your Story' initiative.

However, the FHP approach is not sustainable for the RCP to resource alone. While it was effective pump-priming to deliver demonstration sites and shared evaluations, other approaches need to be explored to facilitate professional-led, 'bottom up' innovation, co-produced with patients working to RCP recommendations for quality improvement, evaluation and innovation. This requires a less formalised and high-investment environment for it to be sustainable in the longer term.

> Patients with access to telemedicine were able to receive specialist care in the community, which resulted in reduced travel time and costs for both patients and physicians.

> Frail, older patients given enhanced community assessment, experienced a reduction in admissions to hospital due to falls.

Another successful part of the programme is the chief registrar scheme, which created a new leadership position for trainee physicians for a minimum of 12 months and determined the skills, protected time and training needed to support them in this role. 16 organisations recruited chief registrars in the pilot first year to develop initiatives that tackle their hospital's critical challenges. By working across teams to address issues such as patient flow and patient safety, chief registrars delivered better outcomes for patients and contributed to improved organisational performance.

An external evaluation of the scheme found that chief registrars had made significant contributions to service improvement leading to increased patient satisfaction, improved patient safety and reduced waiting times. In its second year, more hospital trusts and organisations have recruited chief registrars and the scheme will continue to operate within the RCP's Quality Improvement Programme.

The Future Hospital Programme demonstrated beyond doubt the value of both small and large investments for improvement projects. - Jane Dacre, president, Royal College of Physicians

\section{Quality improvement and the RCP}

The FHP has demonstrated the need for the RCP to support service improvement much more widely, in a way which is which is patientcentred and physician-led. The publication of Delivering the future hospital marked the end of the FHP - however, its legacy will be a RCP faculty to promote, develop and help deliver NHS-wide quality improvement.

The RCP Quality Improvement Programme will build on the considerable learning of FHP, to support physicians and their teams deliver improvements in services and the quality of patient care. Important components of this will be upskilling clinicians in measurement for improvement, facilitating collaborative learning, and supporting patients and carers to be effective members of improvement teams.

The Quality Improvement Programme will provide training, develop learning networks and deliver expert coaching. This programme will complement existing expertise within the RCP including national clinical audit, accreditation, guideline publication and the use of health informatics and digital technology. A major focus will be the development of the next generation of clinical leaders through expansion and refinement of the chief registrar programme. As the RCP approaches its 500-year anniversary in 2018, the FHP has confirmed the RCP is uniquely placed to support physicians lead improvements in the care of their patients.

PROF FRANK JOSEPH Future Hospital officer

Consultant in diabetes and endocrinology and clinical director, Urgent Care Division, Countess of Chester Hospital NHS Foundation Trust

DR MARK TEMPLE Future Hospital officer Consultant in nephrology and general medicine, Heart of England NHS Foundation Trust

\section{Reference}

1 Future Hospital Commission. Future Hospital: caring for medical patients. A report from the Future Hospital Commission to the Royal College of Physicians. London: Royal College of Physicians, 2013. 